\begin{tabular}{|c|c|}
\hline Title & $\begin{array}{l}\text { Femtosecond Carrier Relaxation Dynamics and Photoinduced Phase Separation in } \mathrm{k}-(\mathrm{BEDT}-\mathrm{TTF}) 2 \mathrm{Cu}[\mathrm{N}(\mathrm{CN}) 2] \mathrm{X}(\mathrm{X} \\
=\mathrm{Br}, \mathrm{Cl})\end{array}$ \\
\hline Author(s) & Toda, Y.; Mertelj, T.; Naito, T.; Mihailovic, D. \\
\hline Citation & $\begin{array}{l}\text { Physical Review Letters, 107(22), } 227002 \\
\text { https://doi.org/10.1103/PhysRevLett.107.227002 }\end{array}$ \\
\hline Issue Date & 2011-11-25 \\
\hline Doc URL & http:/hdl.handle.net/2115/47741 \\
\hline Rights & @ 2011 A merican Physical Society \\
\hline Type & article \\
\hline File Information & PRL107-22_227002.pdf \\
\hline
\end{tabular}

Instructions for use 


\title{
Femtosecond Carrier Relaxation Dynamics and Photoinduced Phase Separation in $\kappa-(\mathrm{BEDT}-\mathrm{TTF})_{2} \mathrm{Cu}\left[\mathrm{N}(\mathrm{CN})_{2}\right] X(X=\mathrm{Br}, \mathrm{Cl})$
}

\author{
Y. Toda, ${ }^{1,2}$ T. Mertelj, ${ }^{1}$ T. Naito, ${ }^{3,4}$ and D. Mihailovic ${ }^{1}$ \\ ${ }^{1}$ Complex Matter Department, Jozef Stefan Institute, Jamova 39, Ljubljana, SI-1000, Slovenia \\ ${ }^{2}$ Department of Applied Physics, Hokkaido University, Sapporo 060-8628, Japan \\ ${ }^{3}$ Division of Chemistry, Graduate School of Science, Hokkaido University, Sapporo 060-0810, Japan \\ ${ }^{4}$ Graduate School of Science and Engineering, Ehime University, Matsuyama, 790-8577, Japan
}

(Received 4 August 2011; published 22 November 2011)

\begin{abstract}
We investigate the relaxation dynamics of nonequilibrium carriers in organic conductors $\kappa$-(BEDT-TTF $)_{2} \mathrm{Cu}\left[\mathrm{N}(\mathrm{CN})_{2}\right] X(X=\mathrm{Br}$ and $\mathrm{Cl})$ using ultrafast time-resolved optical spectroscopy. The dynamics for both salts show similar temperature dependences, which is well characterized by the carrier relaxation across the pseudogap (PG) of the magnitude $\Delta_{\mathrm{PG}} \approx 16 \mathrm{meV}$ for $\mathrm{Br}$ salt and $7.0 \mathrm{meV}$ for $\mathrm{Cl}$ salt. On the other hand, only the $\mathrm{Br}$ salt shows an abrupt increase of the decay time at low temperature, indicating an additional decay component associated with the superconducting (SC) gap below $T_{c}$. The fluence dependent dynamics at low temperature evidences the superposition of the SC component onto the PG component. These results indicate a metallic-insulating phase separation in the $\mathrm{Br}$ salt triggered by photoexcited nonequilibrium carriers.
\end{abstract}

DOI: 10.1103/PhysRevLett.107.227002

PACS numbers: 74.70.Kn, 71.30.+h, 78.47.jg

Investigations of nonequilibrium carrier dynamics in strongly correlated electron systems (SCES) have provided new insights into the underlying physics of the correlation and the interactions driving the phase transition. Timeresolved optical pump-probe technique is one of the major experimental techniques to measure such dynamics, where the nonequilibrium distributions of carriers and phonons are induced by an intense pump pulse and their relaxation is traced by a weak probe pulse after the pump pulse. Owing to the high sensitivity in the time domain, the technique allows us to resolve the electronic state stationary points and band extrema near the Fermi energy $\left(E_{F}\right)$. Recently, the framework for analyzing the transient data has being elucidated for various SCES such as high- $T_{c}$ superconductors (SCs) [1-3], heavy fermions [4-6], transition metal oxides [7], and other related compounds [8]. The application to organic SCES is especially interesting because of their rich variety of electronic ground states which can be tuned by (chemical and/or physical) pressure, temperature, and external fields including photoexcitation [9-13]. Since such variety of electronic ground states leads to a complexity of the transient data even within the short time, none of the previous reports have yet been successful in identifying the relaxation processes.

In this Letter, we report the relaxation dynamics of photoexcited carriers in organic molecular salts $\kappa$-(BEDT-TTF $)_{2} \mathrm{Cu}\left[\mathrm{N}(\mathrm{CN})_{2}\right] X$ [BEDT-TTF denotes bis(ethylenedithio)tetrathiafulvalene and $X$ is a counteranion] using the optical pump-probe technique. We compare the dynamics of an isostructural pair of organic salts, $X=\mathrm{Br}$ and $\mathrm{Cl}$ [Fig. 1(b)], as a function of temperature $(T)$ and photoexcitation fluence $(\mathcal{F})$. Except for the SC phase of $\mathrm{Br}$ salt, $T$-dependent dynamics for the two salts are well characterized by a carrier relaxation across the pseudogap (PG). On the other hand, only the Br salt shows an abrupt increase of the decay time when lowering $T$ below $T_{c}$, indicating an additional decay component associated with the SC phase. $\mathcal{F}$-dependent dynamics at low $T$ evidences the superposition of the SC component onto the PG component. The presence of the PG and its coexistence with SC below $T_{c}$ indicate a metallic-insulating phase separation triggered by photoexcited nonequilibrium carriers.

In the experiments, transient reflectivity changes $(\Delta R / R$, where $\Delta R$ is the reflectivity change upon the photoexcitation) were measured on the $a c$ plane of the single crystals of $\mathrm{Br}$ and $\mathrm{Cl}$ salts [Fig. 1(a)], which were mounted in an optical liquid-He flow cryostat. Samples were cooled slowly at a rate of $<1 \mathrm{~K} / \mathrm{min}$. We employed a coaxially aligned two-color pump-probe technique with an on-axis lens $(f=50 \mathrm{~mm})$, allowing for the precise spatial overlap between the beams. The probe pulse was derived from a $250-\mathrm{kHz}$ Ti:sapphire regenerative amplifier $\left[\operatorname{RegA}, E_{\mathrm{pr}}=1.55 \mathrm{eV}(800 \mathrm{~nm})\right]$ and the pump pulse was from a signal of an optical parametric amplifier $\left[E_{\mathrm{pu}}=\right.$ $0.95 \mathrm{eV}(1300 \mathrm{~nm})]$ pumped by the RegA. The time resolution was $\sim 75 \mathrm{fs}$. The effect of steady state heating is negligibly small due to the low repetition rate of the laser, which improves the precision of the $T$ much better than the measurements using high repetition laser [14]. The fluence of the pump pulse $(\mathcal{F})$ ranged from $\sim 10$ to $\sim 400 \mu \mathrm{J} / \mathrm{cm}^{2}$ while the probe fluence was $\sim 3$ orders of magnitude smaller than the pump. The beam diameters of the pump and probe pulses were measured to be 40 and $30 \mu \mathrm{m}$ at the sample position, respectively. Since the dielectric constants in the near-infrared region are roughly identical in each $\kappa$-type salt, the penetration depths were estimated from 
(a)

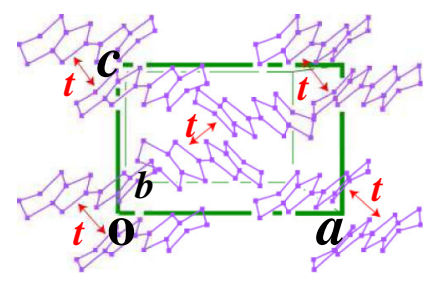

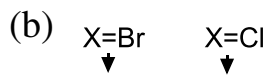
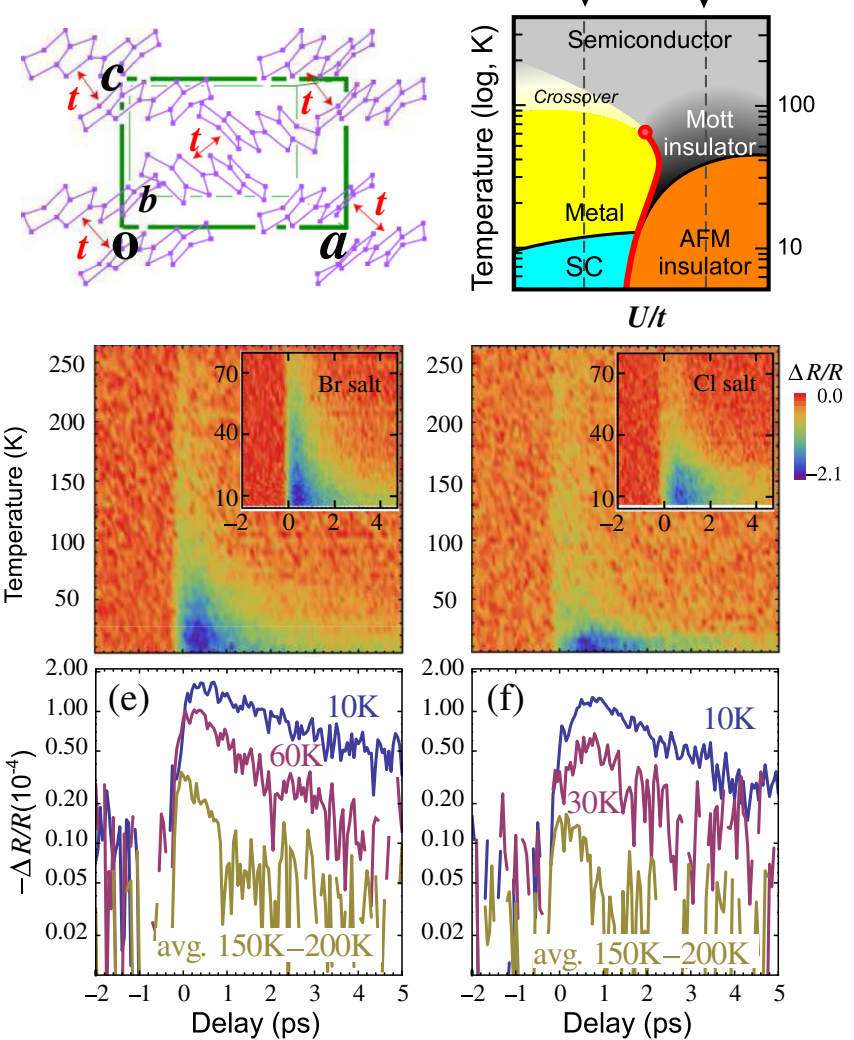

2.00

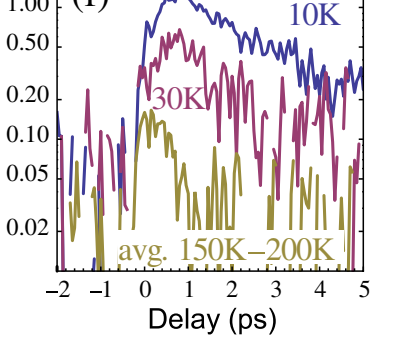

FIG. 1 (color online). (a) The crystal structure of $\kappa$-(BEDT-TTF $)_{2} X$. (b) A schematic phase diagram of $\kappa$-(BEDT-TTF) ${ }_{2} X$ as a function of Coulomb interaction $U / t$. The on-site Coulomb repulsion $U$ is nearly constant for various anions $X$. On the other hand, the substitution of $X$ changes the transfer integral $t$ between BEDT-TTF dimers. AFM denotes antiferromagnetic. (c),(d) Density plots of $\Delta R / R$ transients as a function of $T(5-265 \mathrm{~K}$ with $5 \mathrm{~K}$ step) for $\mathrm{Br}$ salt $(\mathcal{F}=$ $\left.60 \mu \mathrm{J} / \mathrm{cm}^{2}\right)$ and $\mathrm{Cl}$ salt $\left(\mathcal{F}=96 \mu \mathrm{J} / \mathrm{cm}^{2}\right)$, respectively. Insets show the data obtained at low- $T$ region with high resolution steps. (e),(f) $\Delta R / R$ transients at selected temperatures in (c) and (d), respectively. The vertical axis with a log scale represents a pump-excited reduction $(-\Delta R)$ of reflectivity $(R)$.

$\kappa$-(BEDT-TTF $)_{2} \mathrm{Cu}(\mathrm{SCN})_{2}$ [15] to be $1.4 \mu \mathrm{m}$ for $E_{\mathrm{pu}}$ and $4.4 \mu \mathrm{m}$ for $E_{\mathrm{pr}}$.

In Figs. 1(c)-1(f), we plot the $T$ dependence of $\Delta R / R$ transients for (left) $\mathrm{Br}$ and (right) $\mathrm{Cl}$ salts. Figures 1(c) and 1(d) show the density plots of the data, and their cross-sectional views at the selected temperatures are shown in 1(e) and 1(f), respectively. At the conditions of the $E_{\mathrm{pu}} / E_{\mathrm{pr}}=0.95 / 1.55 \mathrm{eV}$ and the probe polarization $\mathbf{E}_{\mathrm{pr}} \| c$, each signal shows a reduction of $R(-\Delta R / R)$. No remarkable polarization dependence was observed in $\mathrm{Cl}$ salt while in $\mathrm{Br}$ salt the amplitude of $\Delta R / R$ becomes small by rotating the polarization away from the $c$ axis. A small increase of $R$ was observed in $\mathrm{Br}$ salt at $\mathbf{E}_{\mathrm{pr}} \| a$ at low temperatures. For simple comparison, we confine ourselves to the data obtained at $\mathbf{E}_{\mathrm{pr}} \| c$, where $\Delta R / R$ in (a)
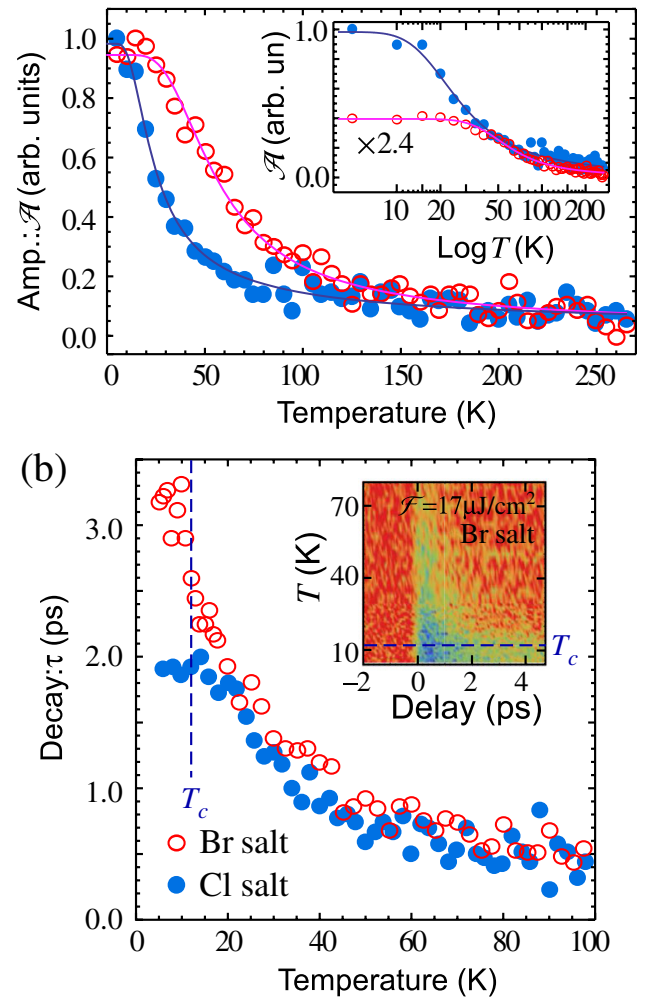

FIG. 2 (color online). $\quad T$ dependences of the $\Delta R / R$ amplitudes $(\mathcal{A})$ (a) and decay times $(\tau)$ (b). Open and closed circles are the data of $\mathrm{Br}$ and $\mathrm{Cl}$ salts, respectively. The solid lines in (a) are fits using $T$-independent gap [3]. To emphasize the similarity of $T$-dependent $\mathcal{A}$ between the salts, the same data are displayed as a function of $\log T$ in the inset of (a), where the data for $\mathrm{Br}$ salt are reduced by 2.4. Inset of (b) shows the density plot for $\mathrm{Br}$ data at low $\mathcal{F}\left(\mathcal{F}=17 \mu \mathrm{J} / \mathrm{cm}^{2}\right)$.

Br salt exhibits the same signature (reduction of $R$ ) as in Cl salt.

For quantitative analysis, $T$ dependences of the amplitude $(\mathcal{A})$ and decay time $(\tau)$ of $\Delta R / R$ are summarized in Fig. 2, where open and solid circles represent the data for the $\mathrm{Br}$ and $\mathrm{Cl}$ salts, respectively. We extracted the maximum value of $\Delta R / R(t)$ for the amplitude $\mathcal{A}$ and normalize it by $\mathcal{A}(T \rightarrow 0)$ [Fig. 2(a)]. The decay time $\tau$ was obtained by fitting the data [insets of Figs. 1(c) and 1(d)] with a single-exponential decay function [Fig. 2(b)]. At $T \gg$ $100 \mathrm{~K}, \Delta R / R$ in each salt is nearly identical and shows an instantaneous transient response, whose $\tau$ is as fast as $\sim 0.5$ ps. Upon lowering $T$ below $\sim 100 \mathrm{~K}, \mathcal{A}$ for both salts shows a gradual increase together with a slight increase of $\tau$. Further lowering of $T$ accelerates these trends and shows plateaus around the lowest $T$. Over the whole $T$ range, $T$ dependence of $\mathcal{A}$ is quite similar between the salts, which is more clearly shown in the inset of Fig. 2(a), where we optimized the normalization of $\mathcal{A}$ to enhance the similarity and plot them as a function of $\log T$. On the other hand, only $\mathrm{Br}$ salt shows an abrupt change of $\tau$, 
which occurs at the SC transition temperature $T_{c} \approx 12 \mathrm{~K}$ [Fig. 2(b)]. In the corresponding $T$ region, the $\mathrm{Cl}$ salt shows a plateau. Except for this difference, $\tau(T)$ is indistinguishable between the salts.

From its $T$ dependence, the additional increase of $\tau$ for $\mathrm{Br}$ salt can be assigned to the SC gap formation. The presence of the SC dynamics is also manifested by the pump fluence $\mathcal{F}$ dependence. In Fig. 3, we summarize $\mathcal{F}$ dependences of $\Delta R / R$ at $T=5 \mathrm{~K}$ for Br salt and $T=10 \mathrm{~K}$ for $\mathrm{Cl}$ salt, respectively. As shown in Figs. 3(c) and 3(d), the relaxation time $\tau$ is strongly $\mathcal{F}$ dependent in $\mathrm{Br}$ salt while only weak $\mathcal{F}$ dependence is seen in $\mathrm{Cl}$ salt, each of which is consistent with the observed $T$ dependence of $\tau$. Moreover, in Fig. 3(a), the deviation from the single-exponential decay is apparent for the $\mathrm{Br}$ salt, indicating the presence of the SC component with a long decay time. To further clarify the component, another density plot of $\Delta R / R$ at the lowest $\mathcal{F}$ is shown in the inset of Fig. 2(b). Although the signal-to-noise ratio is relatively low, which cannot allow the precise estimation of $\mathcal{A}$ and $\tau$, we find a distinct change of $\Delta R / R$ at $T_{c}$, where the long-lived decay component emerges.

The $\mathcal{F}$ dependence of $\mathcal{A}$ is shown in the insets of Fig. 3(c) (Br salt) and 3(d) (Cl salt). For the $\mathrm{Br}$ salt, we also extracted the amplitude of the slow component $\left(\mathcal{A}_{s}\right.$, triangle) by assuming the relaxation with a infinite decay time. With increasing $\mathcal{F}, \mathcal{A}$ for each component first increases linearly, and then deviates from the linear $\mathcal{F}$ dependence. The latter is a signature of saturation and
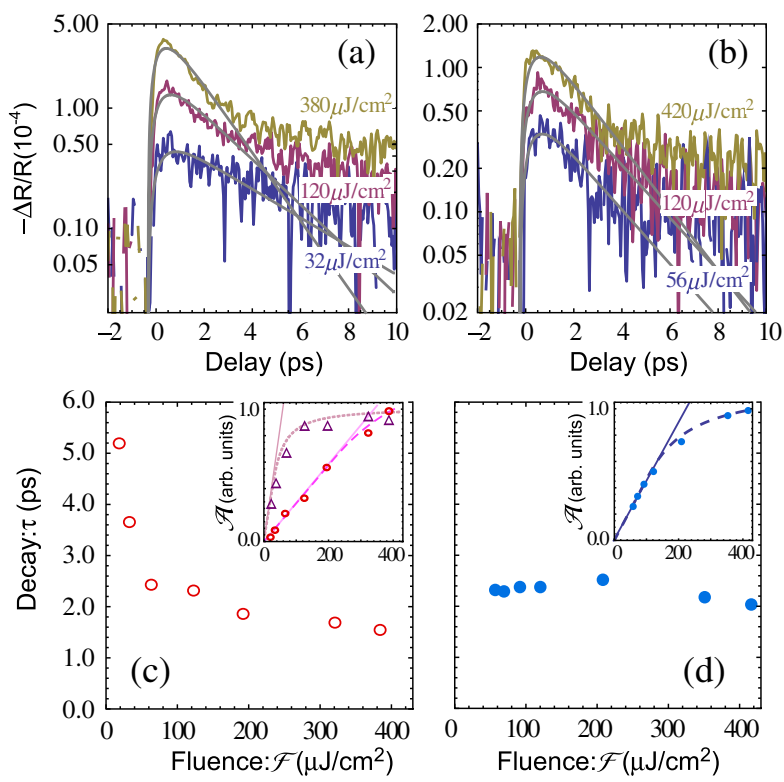

FIG. 3 (color online). $\Delta R / R$ transients at low $T$ excited by various $\mathcal{F}$ for (a) $\mathrm{Br}(T=5 \mathrm{~K})$ and (b) $\mathrm{Cl}(T=10 \mathrm{~K})$ salts. (c), (d) $\mathcal{F}$ dependences of $\tau$ (main panels) and $\mathcal{A}$ (insets). The data were obtained by fits of single-exponential functions [see in (a) and (b)]. Triangles in the inset of (c) show $\mathcal{A}$ for the long-lived component. can be associated with the smearing of the gap [2]. The saturation fluence for $\mathcal{A}_{s}$ is much lower than that of the fast decay component, which is consistent with the restricted density of states for the SC condensation. After the saturation, the fraction of the SC component to $\Delta R / R$ becomes small as compared to the fast decay component with higher saturation value. As a result, the fast decay component becomes dominant in $\Delta R / R$ while the longlived SC component increases $\tau$ with decreasing $T$ below $T_{c}$, as shown in Fig. 2(b).

We now turn to consider the relaxation dynamics of the fast decay component for $\mathrm{Br}$ salt that corresponds to the single decay component for $\mathrm{Cl}$ salt. The monotonic increases of $\mathcal{A}$ and $\tau$ with decreasing $T$, as is observed in both salts, has been ascribed to the PG nature in the context of the phonon mediated relaxation process $[1,3,8]$, where the Kabanov model allows one to check the consistency of the process and to evaluate the magnitude of the gap. In the model, the signal amplitude $\mathcal{A}$ in the weak photoexcitation regime, which should be proportional to a photoexcited population of carriers $n_{\mathrm{pe}}$, is given by[3]

$$
n_{\mathrm{pe}} \propto \mathcal{A}(T)=\left[1+B \exp \left(-\frac{\Delta_{\mathrm{PG}}}{T}\right)\right]^{-1},
$$

where $B$ is a variable constant for the fit and we assume a $T$-independent gap $\Delta_{\mathrm{PG}}$. The weak photoexcitation condition is justified by the fact that $\mathcal{F}$ is well below the saturation condition. The solid lines in Fig. 2(a) show the best fits to the data in each salt, where the magnitudes of the gap are found to be $\Delta_{\mathrm{PG}} \approx 16 \mathrm{meV}$ for the $\mathrm{Br}$ salt and $7.0 \mathrm{meV}$ for the $\mathrm{Cl}$ salt. We stress here that the $T$-dependent dynamics characterized by PG are ascribed to the fast decay component for $\mathrm{Br}$ salt, and at $T<T_{c}$ there also exists the long-lived SC component, i.e., the coexistence of SC and the PG.

We first discuss the PG component in the $\mathrm{Cl}$ salt. The previous optical conductivity measurements have suggested the existence of the PG in this compound, which is composed of the Hubbard bands and connected to the semiconducting nature at higher $T[16,17]$. The optical conductivity spectra also suggested an opening of the $T$-dependent gap associated with the Mott-insulator transition when lowering $T$ below $50 \mathrm{~K}$. The magnitude of the $T$-dependent gap derived from the lowest spectral weight was estimated to be on the order of $100 \mathrm{meV}$, which is much larger than $\Delta_{\mathrm{PG}}$ obtained from our transient data. However, this difference is accounted for by the fact that the optical conductivity measurements probe the direct gap while the pump-probe measurements include the sensitivity to the indirect gap.

Next we discuss the results of $\mathrm{Br}$ salt: $T$-dependent dynamics characterized by PG and coexistence of SC and PG below $T_{c}$. For $\mathrm{Br}$ salt, the presence of any type of gap has not been assigned by the optical conductivity measurements, in which a Drude-like coherent peak obscures the 
spectral shape around $E_{F}$ at low $T[12,16]$. On the other hand, it has been observed by various measurements that a rapid cooling of $\mathrm{Br}$ salt gives rise to a phase separation between metal and insulator [18-20]. Such phase separation is consistent with the PG in our transient data and also with the coexistence with the SC component below $T_{c}$. It has also been suggested that the phase separation is due to a glasslike structural transition at $T_{g} \approx 80 \mathrm{~K}$ [20]. In this case, the cooling rate across $T_{g}$ strongly affects both the metal and SC properties in Br salt. In optical pump-probe measurements, the initial pump pulse excites nonequilibrium carriers in excited states, which is virtually equivalent to an increase of the electronic temperature. The carriers immediately relax down to the states near $E_{F}$ via electronelectron and electron-phonon scattering, resulting in a quasiequilibrium condition of carrier and phonon populations. These processes are completed within the rise time of $\Delta R / R$, which is estimated to be $\sim 0.3$ ps [see Fig. 1(e)]. Therefore, the situation close to the rapid cooling could be realized through the nonequilibrium carrier relaxation.

Although the connection between the nonequilibrium carriers and structural transition is unclear, the structural transition itself is suggested to be governed by either the terminal ethylene groups of BEDT-TTF molecules or the polymetric anion chains $X[20,21]$. Since our pump energy of $E_{\mathrm{pu}}=0.95 \mathrm{eV}$ corresponds to the intramolecular or intradimer transition, the photoexcited carriers can induce a configuration change of the BEDT-TTF molecules, leading to a photoinduced phase separation. Indeed, the specific heat measurement has revealed a reduction of $T_{c}$ with increasing the cooling rate, suggesting that the incomplete structural transition induces a negative pressure, which shifts the system towards the antiferromagnetic state [18]. This is evidenced by the similar trend achieved by the deuteration of $\mathrm{Br}$ and is consistent with our result that $T$-dependent $\mathrm{PG}$ in $\mathrm{Br}$ salt is almost identical to that observed in $\mathrm{Cl}$ salt. Here we note that no remarkable reduction of $T_{c}$ was observed in our nonequilibrium measurement. Since the phase separation induced by nonequilibrium carriers is restricted within the photoexcited volume, the ordered SC phase will be recovered at least within a repetition time of the pulse sequence ( $4 \mu \mathrm{s})$. In the same manner, the equilibrium phase above $T_{c}$ is expected to be metallic before the pump pulse excitation. It is important to note that the photoinduced insulator to metal transition has been reported for the deuterated-Br salt, where the transition is suggested to occur due to intradimer molecular displacement induced by the intradimer excitation [13]. Although the spectral change observed for the deuterated-Br salt indicates that the photoexcitation acts as a positive pressure, which is opposite to our case, it is possible to consider that the similar intradimer molecular displacement stimulates the phase separation for the hydrogenated $\mathrm{Br}$ that initially belongs to the metallic phase.
Finally, we comment on the coexisting PG and SC states for $\mathrm{Br}$ salt. The coexistence of the two gaps has been observed in various high- $T_{c}$ superconductors $[1,2,6,8]$, and two possible origins have been suggested: spatial phase separation and/or two gaps at different points in $k$ space. Here the structural disorder obtained by the rapid cooling process is argued to be responsible for the former. However, it is important to note that band structure calculations predict bands crossing $E_{F}$ at different points in $k$ space [22].

In summary, we have studied the relaxation dynamics of photoexcited carriers in $\kappa$-type BEDT-TTF salts. The dynamics for an isostructural pair of the salts, $X=\mathrm{Br}$ and $\mathrm{Cl}$, show a similar $T$ dependence, which is well characterized by the relaxation bottleneck due to the presence of the PG with $\Delta_{\mathrm{PG}} \approx 16 \mathrm{meV}$ for the $\mathrm{Br}$ salt and $7.0 \mathrm{meV}$ for the $\mathrm{Cl}$ salt. Below $T_{c}$ we found an additional decay component in the $\mathrm{Br}$ salt, which is associated with the $\mathrm{SC}$ phase, indicating the coexistence of the SC and PG phases. The coexistence suggests the presence of the metallic-insulating phase separation in the $\mathrm{Br}$ salt as a consequence of the photoexcitation.

This work was supported by the JSPS Excellent Young Researchers Overseas Visit Program. We would like to thank V. V. Kabanov for fruitful discussions.

[1] J. Demsar et al., Phys. Rev. Lett. 82, 4918 (1999).

[2] P. Kusar et al., Phys. Rev. Lett. 101, 227001 (2008).

[3] V. V. Kabanov et al., Phys. Rev. B 59, 1497 (1999).

[4] J. Demsar et al., Phys. Rev. Lett. 91, 027401 (2003); K. H. Ahn et al., Phys. Rev. B 69, 045114 (2004).

[5] J. Demsar et al., Phys. Rev. Lett. 96, 037401 (2006); J. Demsar et al., Phys. Rev. B 80, 085121 (2009).

[6] D. Talbayev et al., Phys. Rev. Lett. 104, 227002 (2010).

[7] M. Hase et al., Phys. Rev. B 71, 184301 (2005).

[8] T. Mertelj et al., Phys. Rev. Lett. 102, 117002 (2009).

[9] K. Kanoda, J. Phys. Soc. Jpn. 75, 051007 (2006).

[10] P. Limelette et al., Phys. Rev. Lett. 91, 016401 (2003).

[11] F. Kagawa et al., Nature (London) 436, 534 (2005).

[12] J. Merino et al., Phys. Rev. Lett. 100, 086404 (2008).

[13] Y. Kawakami et al., Phys. Rev. Lett. 103, 066403 (2009).

[14] T. Naito et al., J. Phys. Soc. Jpn. 77, 064709 (2008).

[15] A. Ugawa et al., Phys. Rev. B 38, 5122 (1988).

[16] M. Dumm et al., Phys. Rev. B 79, 195106 (2009); D. Faltermeier et al., Phys. Rev. B 76, 165113 (2007).

[17] K. Kornelsen et al., Solid State Commun. 81, 343 (1992).

[18] O. J. Taylor, A. Carrington, and J. A. Schlueter, Phys. Rev. B 77, 060503(R) (2008).

[19] M. A. Tanatar et al., Phys. Rev. B 59, 3841 (1999).

[20] J. Muller et al., Phys. Rev. B 65, 144521 (2002).

[21] A. U. B. Wolter et al., Phys. Rev. B 75, 104512 (2007).

[22] J. M. Williams et al., Science 252, 1501 (1991); W. Y. Ching et al., Phys. Rev. B 55, 2780 (1997). 